\title{
Perfiles clínicos y hemodinámicos en pacientes con falla cardiaca aguda
}

\author{
Luis Eduardo Calderón ${ }^{a}$, Natalia Satizábal ${ }^{a}$, Erika Andrea Rincón ${ }^{\mathrm{b}}$, Pastor Olaya ${ }^{\mathrm{c}}$, \\ Noel Flórez ${ }^{c}$, Diana Cristina Carrillo ${ }^{\mathrm{a}, \mathrm{b}, \mathrm{d}}$, Mayra Estacio ${ }^{\mathrm{a}}$, Isabela Vergara ${ }^{\mathrm{a}}$, \\ Sebastián Yara ${ }^{a}$, Lucy Rivera ${ }^{c}$, Martín Hernando Ramos ${ }^{\mathrm{b}}$ y Juan Esteban Gómez ${ }^{\mathrm{c}, *}$ \\ a Universidad Icesi, Cali, Colombia \\ b Centro de Investigaciones clínicas, Fundación Valle del Lili, Cali, Colombia \\ c Cardiología y Falla Cardíaca, Fundación Valle del Lili, Cali, Colombia \\ ${ }^{\mathrm{d}}$ Fellowship Cardiología, Fundación Valle del Lili, Cali, Colombia
}

Recibido el 1 de diciembre de 2016; aceptado el 25 de abril de 2017

Disponible en Internet el 8 de julio de 2017

\author{
PALABRAS CLAVE \\ Insuficiencia \\ cardíaca; \\ Mortalidad; \\ Agentes inotrópicos
}

\begin{abstract}
Resumen
Objetivo: describir los perfiles clínicos y hemodinámicos de pacientes hospitalizados por falla cardíaca aguda.

Métodos: estudio transversal de pacientes de 18 años o más, con diagnóstico de falla cardíaca aguda admitidos a un centro hospitalario de alta complejidad en Cali, Colombia, en un período de 7 meses. Se recolectó información sociodemográfica, clínica, perfiles clínicos y hemodinámicos de la falla cardiaca al ingreso, estancia hospitalaria y mortalidad. Se describieron las variables cuantitativas como promedios o medianas, y las categóricas como valor absoluto y porcentajes. Se realizaron tablas de frecuencia, y se graficaron los porcentajes de pacientes que ingresaron según el perfil clínico y hemodinámico de falla cardiaca aguda.

Resultados: se incluyeron 99 pacientes con 143 episodios de falla cardíaca aguda. La mitad eran hombres y la mediana de edad 66 años (rango 21 a 97 años). 60\% fueron afrodescendientes y $14,14 \%$ mestizos. Los perfiles clínicos más frecuentes fueron falla cardíaca aguda descompensada $(72,46 \%)$, edema pulmonar agudo $(10,14 \%)$ y falla cardiaca asociada a síndrome coronario agudo en 6,52\%. Los perfiles hemodinámicos fueron "caliente y seco" 10,87\%, "caliente y húmedo"' $55,07 \%$, "frío y húmedo"' $28,26 \%$ y "frío y seco"' en $5,8 \%$.
\end{abstract}

\footnotetext{
* Autor para correspondencia.

Correo electrónico: juanestebang@yahoo.com (J.E. Gómez).
} 


\section{KEYWORDS}

Heart failure; Mortality; Inotropic agents

Conclusiones: la falla cardíaca aguda descompensada es el perfil de presentación clínica más común en nuestra población con dos tercios del total de casos. El perfil hemodinámico más frecuente fue el "caliente y húmedo"' (Stevenson B), resultados que concuerdan con lo observado en estudios locales y registros internacionales.

(c) 2017 Sociedad Colombiana de Cardiología y Cirugía Cardiovascular. Publicado por Elsevier España, S.L.U. Este es un artículo Open Access bajo la licencia CC BY-NC-ND (http:// creativecommons.org/licenses/by-nc-nd/4.0/).

\title{
Clinical and haemodynamic profiles of patients with acute heart failure
}

\begin{abstract}
Objective: To describe the clinical and haemodynamic profiles of patients admitted to hospital due to acute heart failure.

Methods: A cross-sectional study was conducted on patients aged 18 years or older with a diagnosis of acute heart failure admitted to a high complexity hospital in Cali, Colombia, during a 7-month period. Sociodemographic, clinical and haemodynamic information of the cardiac failure was recorded on admission, as well as hospital stay and mortality. The quantitative variables are described as means or medians, and the categorical variables as absolute value and percentages. Frequency tables were made, and a graph made of the percentages of patients admitted, according to the clinical and haemodynamic profile of the acute heart failure.

Results: The study included as total of 99 patients with 143 episodes of acute heart failure. Half of the patients were male, and the median age was 66 years (range 21 to 97 years). The majority $(60 \%)$ were of African descent and $14.14 \%$ were Mestizos. The most common clinical profiles were decompensated heart failure $(72.46 \%)$, acute pulmonary edema $(10.14 \%)$, and heart failure associated with acute coronary syndrome in $6.52 \%$. The haemodynamic profiles were "warm and dry" in 10.87\%, "warm and wet" in $55.07 \%$, "cold and wet" in $28.26 \%$, and "cold and dry" in 5.8\%.

Conclusions: Decompensated acute heart failure is the most common clinical presentation profile in the population studied, with two-thirds of all cases. The most frequent haemodynamic profile was "warm and wet", results that agree with those observed in local studies and in international registers.

(c) 2017 Sociedad Colombiana de Cardiología y Cirugía Cardiovascular. Published by Elsevier España, S.L.U. This is an open access article under the CC BY-NC-ND license (http:// creativecommons.org/licenses/by-nc-nd/4.0/).
\end{abstract}

\section{Introducción}

La falla cardiaca es una de las enfermedades más importantes en el mundo por su alto índice de morbilidad, mortalidad y costo ${ }^{1-3}$. Su epidemiología ha sido bien estudiada, pero su etiología y características clínicas varían por la heterogeneidad de las poblaciones ${ }^{1,4}$. La prevalencia de esta condición en Europa y Norteamérica es de 1 a $2 \%$ y se estima que aumentará de forma simultánea con el descenso de la mortalidad por enfermedad coronaria y cerebrovascular ${ }^{1,5,6}$. En Colombia, para el año 2012, la prevalencia fue 2,3\%, es decir, 1'100.000 de colombianos padecían la enfermedad ${ }^{7}$.

De los individuos diagnosticados con falla cardiaca, un millón por año presentarán hospitalizaciones en el contexto de un episodio de falla cardiaca aguda (FCA). Cerca de $45 \%$ serán una rehospitalización después de un evento agudo, $15 \%$ un segundo evento hospitalario antes del año, y es probable que continúe en aumento por el incremento de la expectativa de vida de la población, el uso de tratamientos que incrementan la sobrevida luego de un infarto del miocardio y los tratamientos con el fin de prevenir la muerte cardíaca súbita ${ }^{1,4,6,8}$.
Estos episodios pueden clasificarse de acuerdo con sus características o perfiles hemodinámicos y clínicos. En el primero se describen estadios según la presencia o ausencia de signos y síntomas de congestión e hipoperfusión (estadio $A, B, C \circ L$ ) y en el segundo la característica clínica más relevante de la descompensación (FCA descompensada, edema agudo de pulmón, crisis hipertensiva, shock cardiogénico y falla cardíaca derecha $)^{2,9-11}$. En este estudio se describen las características clínicas y hemodinámicas de los episodios de FCA en una institución hospitalaria de alta complejidad en Cali, Colombia.

\section{Métodos}

Estudio de corte transversal, basado en el Registro Institucional de FAlla CArdíaca aguda (RIFACA). El registro recolecta información de forma sistemática, prospectiva, a través de Internet, de variables sociodemográficas, antecedentes médicos, síntomas relacionados con el inicio del episodio de FCA, hallazgos al examen físico, paraclínicos y biomarcadores cardiacos, radiológicos y ecocardiográficos, 
perfil clínico y hemodinámico de ingreso, necesidad de medicamentos hospitalarios como vasoactivos, inotrópicos o vasodilatadores, y desenlaces clínicos como mortalidad hospitalaria y rehospitalizaciones. En el registro también se incluyen escalas de calidad de vida y de síntomas relacionados con falla cardiaca. A estos pacientes se les hace un seguimiento en el tiempo cuando hayan pasado 1 , $6,12,24,36,48$ y 60 meses después del alta hospitalaria.

Para este estudio fueron elegibles pacientes que ingresaron al servicio de urgencias, hospitalización y unidad de cuidados intensivos ( $\mathrm{UCl}$ ) de la Fundación Valle del Lili en Cali, Colombia, en un período de 7 meses, desde el 01 de noviembre de 2015 hasta el 31 de mayo de 2016, por síntomas clínicos sugestivos de FCA confirmada mediante biomarcadores cardiacos positivos, dados por la prohormona $\mathrm{N}$-terminal del péptido natriurético (NT-proBNP) $\geq$ $450 \mathrm{pg} / \mathrm{ml}$ en pacientes menores de 50 años, $\geq 900 \mathrm{pg} / \mathrm{ml}$ en pacientes entre 50 y 75 años $\mathrm{y}, \geq 1.800 \mathrm{pg} / \mathrm{ml}$ en adultos mayores de 75 años, o la presencia de alteración estructural o funcional cardíaca concomitante con síntomas ${ }^{1,11,12}$. Se incluyeron pacientes que aceptaron participar mediante firma de consentimiento informado y tuvieron valoración por cardiología durante su estancia hospitalaria. Después del 7 de diciembre de 2015 se realizó una enmienda al protocolo de investigación luego de recolectar los primeros 12 pacientes, en la cual se retiró el consentimiento informado del estudio, permitiendo el ingreso sistemático de pacientes con FCA. Fueron excluidos aquellos en quienes, posterior a la valoración, se confirmó que la causa de los síntomas no estaba relacionada con falla cardíaca.

Se definieron los perfiles clínicos de ingreso de acuerdo con la clasificación de la Sociedad Europea de Cardiología (ESC) del año 2008:

- FCA descompensada (de novo o como descompensación aguda de falla cardiaca crónica) con signos y síntomas de FCA leves y no cumplen los criterios para shock cardiogénico, edema pulmonar o crisis hipertensiva.

- Edema pulmonar como FCA acompañada de dificultad respiratoria, crépitos pulmonares y ortopnea, con saturación de oxígeno $\left(\mathrm{SaO}^{2}\right)<90 \%$ a aire ambiente previo al tratamiento.

- Shock cardiogénico como evidencia de hipoperfusión tisular inducida por falla cardiaca luego de corregir la precarga, con reducción de la presión arterial sistólica (PAS) $<90 \mathrm{~mm} \mathrm{Hg}$ o una caída de la tensión arterial media $>30 \mathrm{~mm} \mathrm{Hg}$ y/o bajo gasto urinario $(<0,5 \mathrm{ml} / \mathrm{kg} / \mathrm{h})$ con un pulso $>60 \mathrm{lpm}$, con o sin evidencia de congestión de órganos.

- FCA hipertensiva como signos y síntomas de falla cardiaca asociados con cifras tensionales altas, y radiografía de tórax compatible con edema agudo de pulmón.

- Falla cardiaca derecha como síndrome de bajo gasto asociado con aumento de la presión venosa yugular, hepatomegalia e hipotensión.

- Asociada a síndrome coronario agudo (SCA) como falla cardiaca con evidencia clínica y paraclínica de SCA ${ }^{1,13,14}$.

Los perfiles hemodinámicos se clasificaron según la presencia o ausencia de signos de hipoperfusión (presión de pulso estrecha, alternancia de pulso, hipotensión sintomática, frialdad de extremidades, alteración en el sensorio, llenado capilar lento, disfunción renal aguda y acidosis láctica) y de congestión (crépitos pulmonares, ortopnea, ingurgitación yugular, reflujo hepatoyugular, ascitis, edema periférico y $\$ 3)^{1,4}$ en:

- Estadio A o como la ausencia de signos de congestión e hipoperfusión ("caliente y seco").

- Estadio B sin signos de hipoperfusión y con signos de congestión ("caliente y húmedo"). Estadio $\mathrm{C}$ definido por presencia de signos de hipoperfusión y signos de congestión ("frío y húmedo").

- Estadio L con signos de hipoperfusión y sin signos de congestión ("frío y seco") )"

\section{Análisis estadístico}

Las variables se clasificaron en grupos como datos demográficos, clínicos (signos y síntomas), antecedentes médicos cardiovasculares como enfermedad coronaria previa, hipertensión arterial (HTA), tromboembolia pulmonar (TEP), entre otros, y no cardiovasculares como falla renal, enfermedad pulmonar obstructiva crónica (EPOC), etc. Se hizo una descripción de la información obtenida. Variables cuantitativas como edad, peso, fracción de eyección, etc., se describieron como promedios o medianas, y desviación estándar o rango intercuartil (RIQ) según la presencia de distribución normal. Para esto se aplicó la prueba de Shapiro-Wilk. Las variables categóricas se describieron como porcentajes. La falla cardiaca se clasificó de acuerdo con la fracción de eyección por ecocardiografía en:

- Falla cardiaca con fracción de eyección reducida, definida como síntomas y signos clínicos de FCA con una fracción de eyección del ventrículo izquierdo $(\mathrm{FEVI})<40 \%$.

- Falla cardiaca con fracción de eyección en rango medio, definida como síntomas y signos clínicos de FCA con una FEVI entre 40-49\%, daño estructural relevante.

- Falla cardiaca con fracción de eyección preservada, definida por síntomas y signos clínicos de FCA con FEVI $\geq$ $50 \%$

Se determinó la prevalencia de perfiles clínicos y hemodinámicos de FCA. Se presentaron estos valores en tablas de frecuencia y se elaboró un gráfico de barras para la prevalencia de estos perfiles de FCA y otro para la mortalidad según perfil clínico y hemodinámico. Para el análisis estadístico se utilizó Stata $14^{\circledR}$. Este estudio tuvo aprobación del Comité de Ética en Investigación Biomédica de la Fundación Valle del Lili y patrocinio de la Universidad Icesi y la Fundación Valle del Lili.

\section{Resultados}

Desde el 01 de noviembre de 2015 hasta el 31 de mayo de 2016 se incluyeron 99 pacientes que presentaron 143 episodios de descompensación de falla cardíaca (fig. 1). Las 


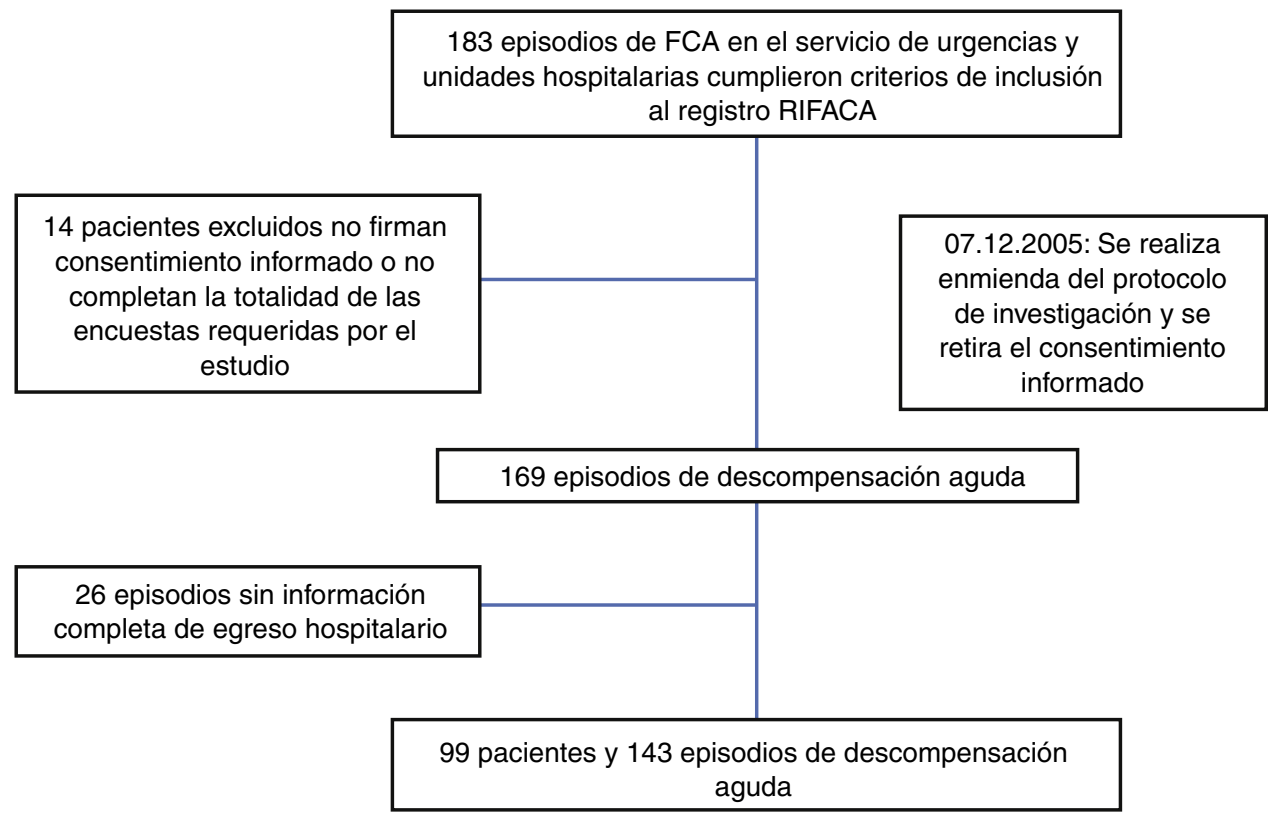

RIFACA: Registro Internacional de Falla Cardiaca Aguda; FCA: Falla cardiaca aguda

Figura 1 Flujograma de pacientes con FCA que consultaron por urgencias o servicios hospitalarios.

características sociodemográficas de la población se muestran en la tabla 1. La mediana de edad fue 66 años (rango intercuartil [RIQ] 55-75 años) y el promedio de 62,57 $\pm 19,50$ años. La mitad fueron de género masculino $(52,52 \%)$. La etnia más prevalente fue la afrodescendiente en $60,61 \%$ seguida por la indígena en $21,21 \%$ de los casos. Cerca de $64 \%$ de los pacientes tenían afiliación al sistema de salud bajo el régimen subsidiado y solo $24 \%$ en régimen contributivo.

Los antecedentes patológicos más frecuentes fueron: hipertensión arterial (55,56\%), diabetes mellitus $(23,23 \%)$, fibrilación auricular $(20,20 \%)$, enfermedad coronaria $(25,25 \%)$ e implante de terapia de resincronización cardíaca con o sin desfibrilador (20\%) (tabla 1). Cerca de un quinto de los individuos reportaron hospitalizaciones por FCA en el año previo (mediana 1 hospitalización, RIQ 1-2). Al ingreso, $62,94 \%$ de los pacientes recibían betabloqueadores, $66,44 \%$ inhibidores de la enzima convertidora de angiotensina (IECA) o antagonistas de receptores de angiotensina ॥ (ARAII), 50,35\% diurético ahorrador de potasio (espironolactona o epleronone) y 64,34\% diuréticos de asa (tabla 2).

La etiología de la falla cardíaca fue isquémica en $32,87 \%$, dilatada idiopática en $20,28 \%$ y valvulopatía en $15,38 \%$. Menos del $10 \%$ de los casos se atribuyeron a causas congénitas, chagásica, restrictiva, hipertrófica e hipertensiva. Los principales eventos desencadenantes de los episodios de FCA fueron en el contexto de una cirugía cardíaca $(20,98 \%)$, desconocida $(18,18 \%)$, no adherencia a fármacos $(15,38 \%)$ y arritmias $(11,89 \%)$ (tabla 3$)$. El principal síntoma referido al ingreso fue disnea en $68,53 \%$ de los casos y $79,02 \%$ de los pacientes se encontraban en clase funcional New York Heart Association (NYHA) III O IV.

Los hallazgos al examen físico de ingreso y ayudas diagnósticas utilizadas se exponen la tabla 4. La mediana de fracción de eyección fue 30\% (RIQ 16-55\%) y 30\% tenían FEVI preservada, $5,56 \%$ en rango medio y $64,44 \%$ FEVI reducida.
El perfil clínico al ingreso hospitalario más frecuente fue la falla cardiaca crónica con descompensación aguda $(72,46 \%)$ seguido de FCA con edema pulmonar. Solo $2,17 \%$ de los pacientes tuvieron perfil clínico de ingreso de shock cardiogénico (fig. 2). El perfil hemodinámico más frecuente fue el estadio $B$ en $55,07 \%$ seguido de estadio $C$ en $28,26 \%$ y por último estadios $A$ y $L$ en $10,87 \%$ y $5,8 \%$ respectivamente (fig. 3). En concordancia con lo anterior, los medicamentos endovenosos utilizados para el manejo del episodio de FCA fueron furosemida $72 \%$, seguido de milrinone $40,5 \%$, nitroglicerina $24,4 \%$ y norepinefrina $21,6 \%$ (fig. 4).

De los episodios de FCA del estudio, 67,1\% fueron admitidos a UCI con una mediana de estancia de 5 días (RIQ 3-7 días). La ventilación mecánica invasiva fue necesaria en $16 \%$ y la ventilación mecánica no invasiva en $18,8 \%$. Tanto el implante de resincronizador cardiaco como el de desfibrilador se requirió en $4,9 \%$ de los pacientes. La mortalidad hospitalaria global fue $12,12 \%$, sin diferencias de acuerdo con el perfil clínico o hemodinámico al ingreso (figs. 5 y 6). La mediana de estancia hospitalaria fue 6 días (RIQ 3-11 días).

\section{Discusión}

Los eventos hospitalarios por FCA son predictores de mortalidad y de reingresos hospitalarios ${ }^{4}$. La población del mundo es heterogénea respecto a las características sociodemográficas y clínicas de los pacientes con FCA e inclusive puede variar en regiones dentro del mismo país y en diferentes períodos. En Colombia, se identificaron tres artículos publicados que incluyen pacientes con FCA y con similitudes respecto a la distribución de edad y género, pero, con diferencias en la etiología, factores precipitantes y mortalidad $^{15,17}$. El primero, publicado en 2004 por Ospina et al. (tabla 5), incluyó 218 pacientes con FCA en un período 


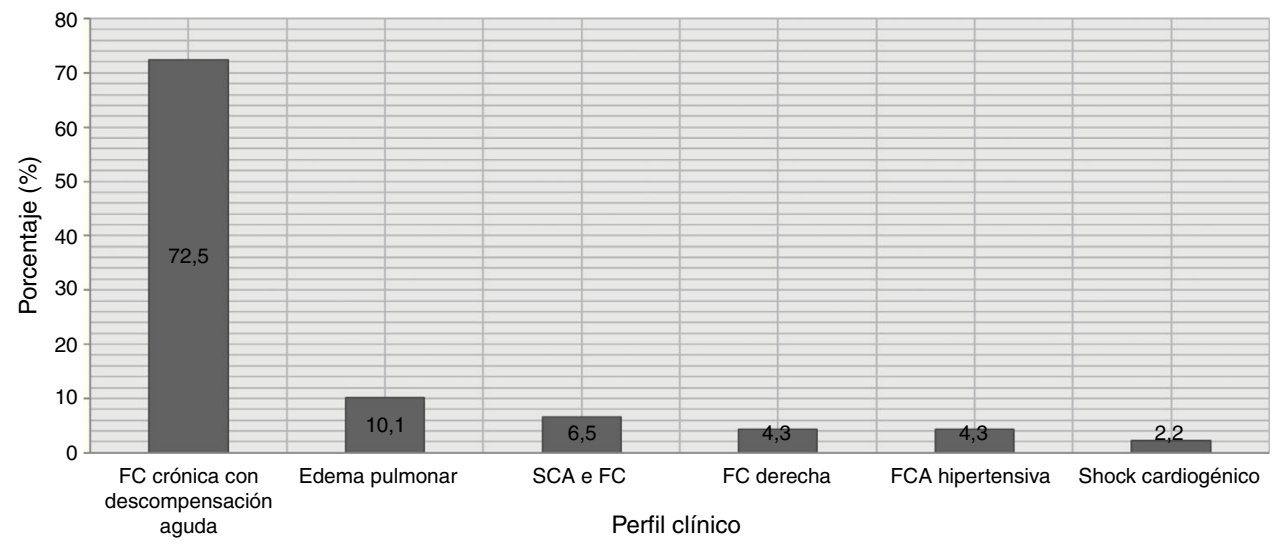

FC: Falla cardiaca; FCA: Falla cardiaca aguda: SCA: Síndrome coronario agudo

Figura 2 Perfiles clínicos de los episodios de falla cardíaca aguda al ingreso hospitalario.

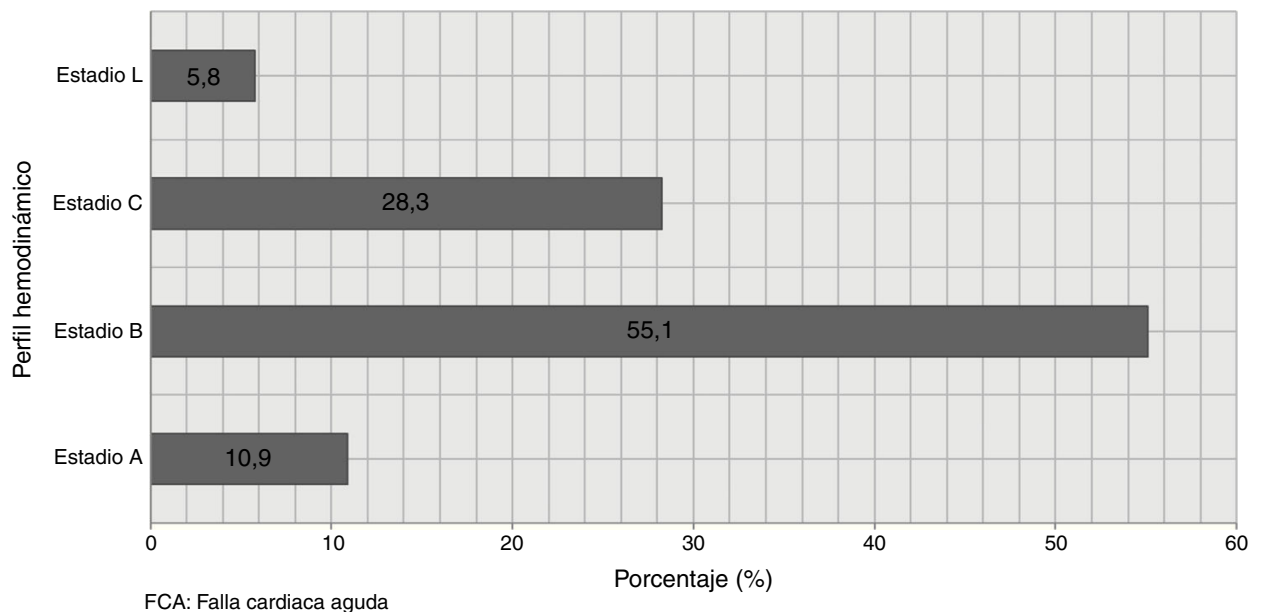

Figura 3 Perfiles hermodinámicos de los episodios de falla cardíaca aguda al ingreso hospitalario.

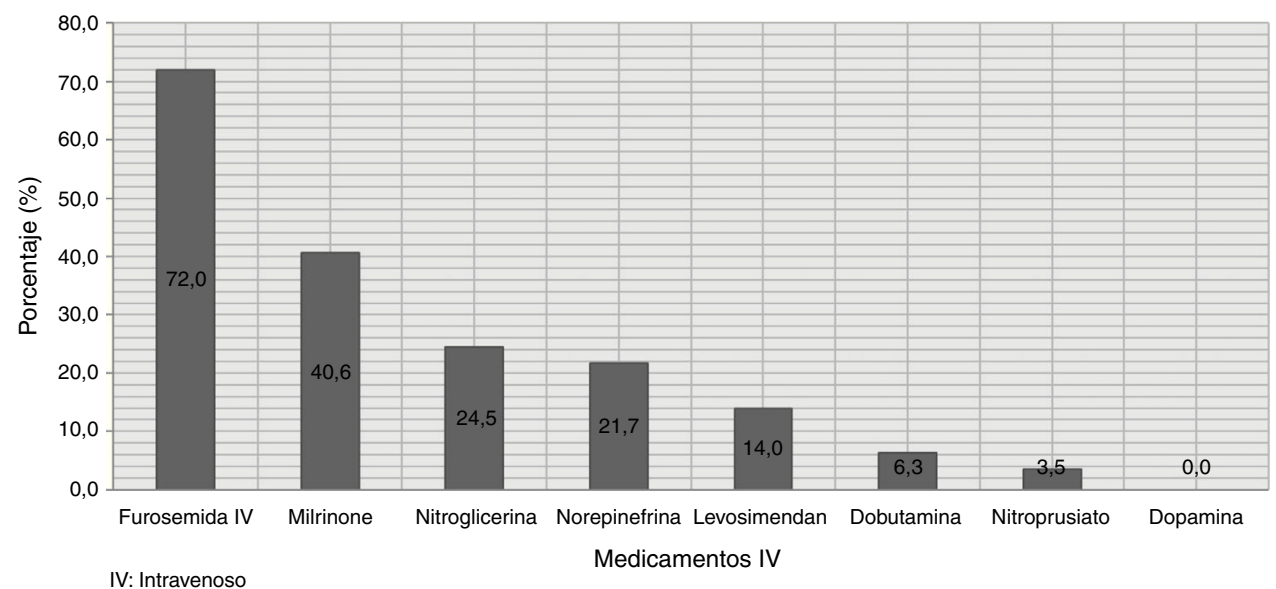

Figura 4 Medicamentos intravenosos utilizados en los episodios de descompensación.

de un año. El promedio de edad fue 68 años y cerca de la mitad de los pacientes eran de sexo masculino. La principal causa de exacerbación de FCA fue la no adherencia al tratamiento médico 50\%, infección respiratoria 15,1\% y exacerbación de EPOC en 11,9\%. La estancia hospitalaria fue más larga comparativamente con nuestros resultados (10 vs. 6 días respectivamente) así como también lo fue la mortalidad (16,9\% vs. $12,12 \%)$. Ospina et al. describieron solo $6 \%$ de pacientes que recibían IECA y betabloqueadores al ingreso vs. $43,35 \%$ descrito en el presente documento. 


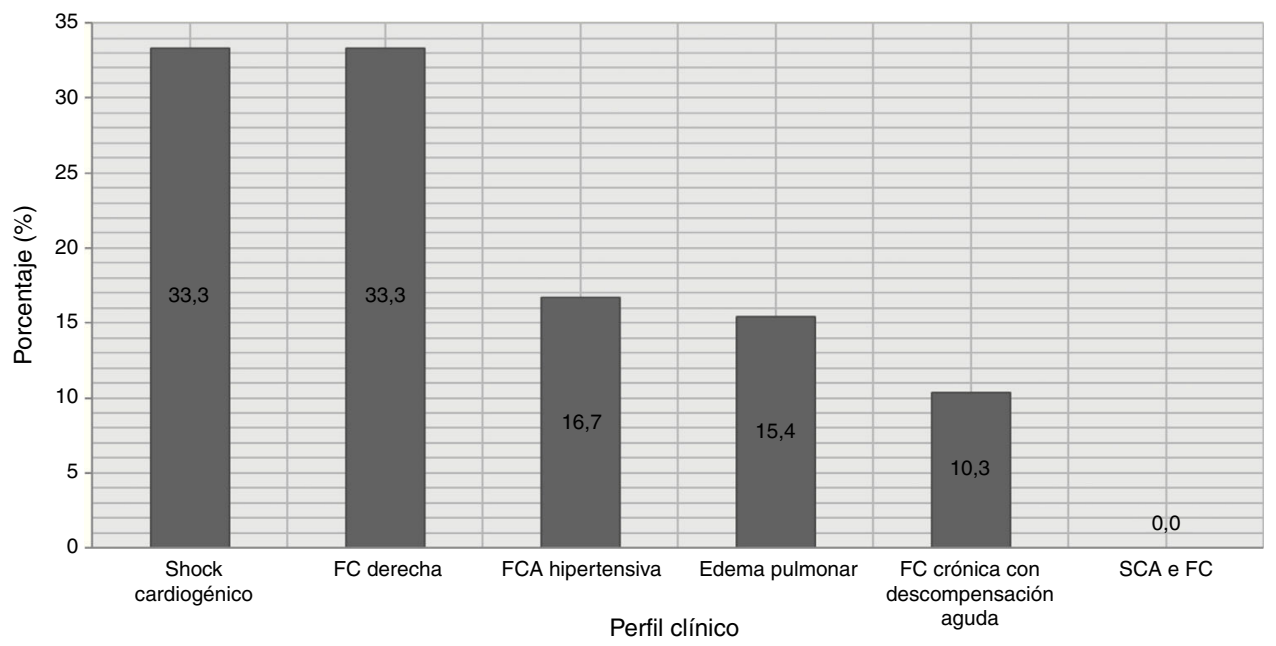

FC: Falla cardiaca; FCA: Falla cardiaca aguda; SCA: Síndrome coronario agudo

Figura 5 Mortalidad hospitalaria de acuerdo con el perfil clínico de ingreso.

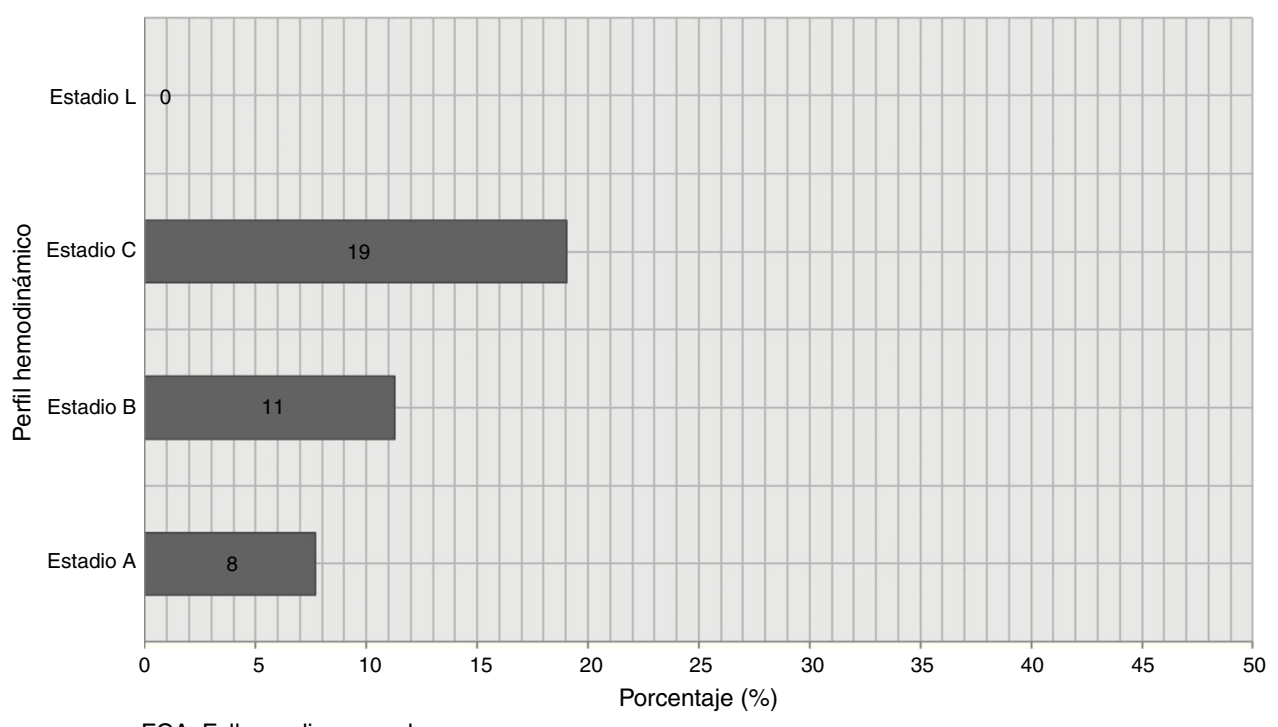

FCA: Falla cardiaca aguda

Figura 6 Mortalidad hospitalaria según el perfil hemodinámico de ingreso.

A diferencia de lo reportado en nuestro estudio, la EPOC no fue una de las patologías más frecuentes $(9,84 \%$ vs. $20 \%$ respectivamente), al igual que la falta de adherencia al tratamiento médico como causa del ingreso hospitalario $(15 \%$ vs. $50 \%$ ), aunque esta cifra podría estar subestimada. En nuestro estudio, 93 de los 143 episodios tenían ecocardiograma durante la hospitalización de los cuales 60 eventos $(41,95 \%)$ tenían fracción de eyección menor a 40\% comparativamente con lo descrito por Ospina et al. donde $73 \%$ tenían disfunción sistólica, resultado que podría explicar la mayor mortalidad observada en dicho estudio. Sin embargo, en nuestra población la mortalidad entre los que tenían $\mathrm{FEVI}<40 \%$ vs. $\geq 40 \%$ no fue diferente (mortalidad $8,33 \%$ vs. 9,09 respectivamente $p=1,00$ ). Aunque en el estudio de Ospina et al. no describen la etiología de la falla cardíaca, solo $17 \%$ de los pacientes son reportados con cardiopatía isquémica, comparativamente con nuestro registro donde
$32,87 \%$ de los pacientes tienen cardiopatía isquémica y solo 4,2\% hipertensiva ${ }^{15}$.

En el 2010, Castaño et al. (tabla 5) reportaron 370 pacientes con falla cardiaca en un período de 3 años (2005 a 2008) en un primer nivel de atención hospitalaria. El promedio de edad fue 69,59 años (rango 17-96 años) con mayor prevalencia de EPOC comparativamente con nuestro estudio $(31,4 \%$ vs. $11,9 \%)$. No reportaron mortalidad, sin embargo, describen uso de la combinación de IECA y diuréticos en $13,2 \%$ menor a nuestros hallazgos donde se observó $46,15 \%$ del uso de esta combinación. El uso de betabloqueadores descrito por Castaño et al. solo fue de $16,8 \%$ vs. lo reportado en nuestro estudio donde 55,56\% de los pacientes con FEVI disminuida recibían algún betabloqueador al momento del ingreso hospitalario ${ }^{16}$.

Senior et al. (tabla 5) en el año 2011 describieron 106 pacientes con FCA con edad promedio de 62,4 años (rango 
Tabla 1 Características sociodemográficas y antecedentes médicos de los pacientes que ingresaron por falla cardiaca aguda

\begin{tabular}{|c|c|}
\hline Variable & $\mathrm{n}(\%), \mathrm{n}=99$ \\
\hline Edad" & $66(55-75)$ \\
\hline \multicolumn{2}{|l|}{ Género } \\
\hline Femenino & $48(48,48)$ \\
\hline Masculino & $51(51,52)$ \\
\hline \multicolumn{2}{|l|}{ Régimen de salud } \\
\hline Contributivo & $24(24,24)$ \\
\hline Subsidiado & $63(63,64)$ \\
\hline Medicina prepagada & $11(11,11)$ \\
\hline Particular & $1(1,01)$ \\
\hline \multicolumn{2}{|l|}{ Etnia } \\
\hline Mestiza & $14(14,14)$ \\
\hline Afrodescendiente & $60(60,61)$ \\
\hline Indígena & $21(21,21)$ \\
\hline Otro & $2(2,02)$ \\
\hline Sin información & $2(2,02)$ \\
\hline \multicolumn{2}{|l|}{ Antecedentes médicos } \\
\hline Hipertensión arterial & $55(55,56)$ \\
\hline Diabetes mellitus & $23(23,23)$ \\
\hline Dislipidemia & $12(12,12)$ \\
\hline Enfermedad renal crónica & $25(25,25)$ \\
\hline Tabaquismo actual & $22(22,22)$ \\
\hline Enfermedad coronaria & $25(25,25)$ \\
\hline Stent coronario & $11(11,11)$ \\
\hline CABG & $5(5,05)$ \\
\hline Prótesis valvular & $9(9,09)$ \\
\hline Otra cirugía cardíaca & $3(3,03)$ \\
\hline Tromboembolia venosa & 9,09 \\
\hline Ataque cerebrovascular & $9(9,09)$ \\
\hline Fibrilación o flutter auricular & $21(21,21)$ \\
\hline Valvulopatía aórtica & $8(8,08)$ \\
\hline Valvulopatía mitral & $28(28,28)$ \\
\hline Valvulopatía tricúspide & $21(21,21)$ \\
\hline \multicolumn{2}{|c|}{ Dispositivos electrónicos cardiovasculares implantables } \\
\hline Marcapaso & $4(4,04)$ \\
\hline CRT-P & $2(2,02)$ \\
\hline CRT-D & $18(18,18)$ \\
\hline CDI & $4(4,04)$ \\
\hline
\end{tabular}

" Valor expresado en mediana (rango intercuartil), CABG: coronary artery bypass graft en su sigla en inglés, CRT-P: terapia de resincronización cardíaca, CRT-D: terapia de resincronización cardíaca con desfibrilador, CDI: cardiodesfibrilador implantable.

16 a 94 años) y 52,8\% hombres. La etiología de la falla cardíaca reportada fue: isquémica en $14,1 \%, 45,2 \%$ hipertensiva, $31,1 \%$ idiopática, $8,4 \%$ valvular y $11,2 \%$ asociada a SCA, mientras que nuestro estudio muestra un $32,87 \%$ de origen isquémico y $4,2 \%$ hipertensivo. Asimismo, describieron el perfil hemodinámico de ingreso como estadio $\mathrm{B}$ (caliente-húmedo) $96,6 \%$, estadio $C$ (frío-seco) $1,4 \%$, estadio $L$ (frío-húmedo $2,6 \%$ ) proporción menor en estadio $C$ y $L$ a la reportada por nuestro registro $(28,26 \%$ y $5,8 \%$ respectivamente) ${ }^{17}$.
Tabla 2 Tratamiento farmacológico recibido previo al ingreso por falla cardiaca aguda

\begin{tabular}{ll}
\hline Variable & $\mathrm{n}(\%), \mathrm{n}=143$ \\
\hline Aspirina & $38(26,57)$ \\
Inhibidores P2Y12 & $6(4,20)$ \\
Anticoagulantes & $32(22,38)$ \\
Estatinas & $41(28,67)$ \\
Betabloqueadores & $90(62,94)$ \\
IECA/ARA II & $95(66,43)$ \\
Vasodilatadores & $6(4,20)$ \\
Digoxina & $42(29,37)$ \\
Amiodarona & $4(2,80)$ \\
Calcioantagonistas & $21(14,70)$ \\
Diuréticos & $92(64,34)$ \\
Antagonistas de aldosterona & $72(50,35)$ \\
Ivabradina & $3(2,10)$ \\
\hline
\end{tabular}

IECA: inhibidores de la enzima convertidora de angiotensina. ARA ॥: antagonistas del receptor de angiotensina ॥. Diuréticos disponibles en Colombia: furosemida, hidroclorotiazida.

Los perfiles de presentación clínica descritos en las guías europeas son de ayuda para clasificar los pacientes y así tener una noción pronóstica de la mortalidad que pueden presentar $^{13}$. El perfil clínico más frecuente asociado con ingresos a urgencias por FCA fue la falla cardíaca crónica con descompensación aguda $(72,5 \%)$ seguido de edema pulmonar $(10,1 \%)$ y solo $2,17 \%$ tuvieron shock cardiogénico. Los registros internacionales AHEAD (Acute Heart Failure Database) y ALARM HF describen una menor proporción de pacientes de falla cardiaca crónica con descompensación aguda y mayor frecuencia de shock cardiogénico (FCA descompensada $55,3 \%$ y $38,6 \%$, shock cardiogénico $11,7 \%$ respectivamente). A pesar de lo anterior la mortalidad hospitalaria es similar a la descrita en nuestro estudio $(12,7 \%$ vs. $12 \%$ vs. $12,12 \%$ respectivamente) $)^{18-20}$. Sin embargo, el registro ADHERE describió una mortalidad intrahospitalaria menor de 4\% en los pacientes con FCA, aunque alcanzó 10,6\% para aquellos que ingresaron a cuidado intensivo ( $\mathrm{UCl}$ ), comportamiento similar a nuestros hallazgos donde los pacientes que ingresaron a UCI $(67,13 \%)$ tuvieron una mortalidad de $9,38 \%$ vs. $6,38 \%$ en los pacientes que fueron atendidos en las unidades hospitalarias del servicio $(p=0,57)^{21}$. Un subestudio del ADHERE reporta aún menor mortalidad en pacientes con FEVI preservada $(2,8 \%)$ vs. FEVI disminuida $(3,9 \%)^{22}$. Otros registros como EHFS I, EHFS ॥, FINN-AVKA, OPTIMIZEHF reportaron mortalidades de $6,9 \%, 6,7 \%, 7,1 \%$ y $3,8 \%$ respectivamente $e^{2,23-26}$.

Los hallazgos de perfil hemodinámico concuerdan con los de la literatura mundial: los pacientes con FCA en perfiles $B$ y $C$ tienen mayor mortalidad comparativamente con el perfil A (perfil B HR 2,1 95\% IC 1,29-3,43, perfil C HR 3,66 95\% 2,16$6,21)^{10}$. En nuestro estudio los pacientes que ingresaron con perfiles hemodinámicos $B$ y $C$ fueron los que tuvieron mayor mortalidad hospitalaria.

Los medicamentos intravenosos utilizados más frecuentemente fueron los diuréticos (72\%), seguidos de inotrópicos en $50 \%$ y vasoactivos en $20 \%$. El alto uso de inotrópicos contrasta con el bajo porcentaje de perfil hemodinámico C ("frío y húmedo"). Estos hallazgos podrían tener dos 
Tabla 3 Etiología y factores desencadenantes del episodio de falla cardiaca aguda

\begin{tabular}{|c|c|}
\hline Variable & $n(\%), n=143$ \\
\hline \multicolumn{2}{|l|}{ Etiología falla cardiaca } \\
\hline Isquémica & $47(32,87)$ \\
\hline Hipertensiva & $6(4,20)$ \\
\hline Hipertrófica & $1(0,70)$ \\
\hline Dilatada & $29(20,28)$ \\
\hline Restrictiva & $3(2,10)$ \\
\hline Chagásica & $1(0,70)$ \\
\hline Viral & $1(0,70)$ \\
\hline Valvulopatía & $22(15,38)$ \\
\hline Enfermedad pericárdica & $1(0,70)$ \\
\hline Congénita & $4(2,80)$ \\
\hline Otro & $9(6,30)$ \\
\hline Desconocida & $12(8,39)$ \\
\hline Sin información & $7(4,90)$ \\
\hline \multicolumn{2}{|l|}{ Factor precipitante falla cardiaca aguda } \\
\hline SCA & $10(6,99)$ \\
\hline Arritmias & $17(11,89)$ \\
\hline Infección & $9(6,29)$ \\
\hline No adherencia fármacos & $22(15,38)$ \\
\hline No adherencia dieta/líquidos & $9(6,29)$ \\
\hline HTA no controlada & $6(4,20)$ \\
\hline Problemas quirúrgicos/perioperatorios & $2(1,40)$ \\
\hline Exacerbación EPOC/asma & $2(1,40)$ \\
\hline Cirugía cardíaca & $30(20,98)$ \\
\hline Desconocida & $27(18,88)$ \\
\hline Sin información & $9(6,29)$ \\
\hline \multicolumn{2}{|l|}{ Síntomas principal ingreso } \\
\hline Disnea & $98(68,53)$ \\
\hline Edemas & $7(4,90)$ \\
\hline Angina & $15(10,49)$ \\
\hline Síncope & $4(2,80)$ \\
\hline Fiebre & $1(0,70)$ \\
\hline Dolor abdominal & $6(4,20)$ \\
\hline Alteraciones neurológicas & $4(2,80)$ \\
\hline Otro síntoma & $7(4,90)$ \\
\hline Sin información & $1(0,70)$ \\
\hline
\end{tabular}

FC: falla cardíaca, FCA: falla cardíaca aguda. EPOC: enfermedad pulmonar obstructiva crónica.

explicaciones: los pacientes en falla cardiaca terminal que reciben ciclos paliativos de inotrópico con el fin de mejorar síntomas y que probablemente no se clasifican de forma precisa en alguno de los perfiles hemodinámicos de Stevenson, y las descompensaciones agudas en el contexto perioperatorio de cirugía cardíaca o no cardíaca donde el comportamiento hemodinámico es fluctuante cumpliendo con criterios de hipoperfusión y por lo tanto de inicio de medicamentos vasoactivos o inotrópicos.

La principal fortaleza es el registro prospectivo de pacientes con FCA en la era más contemporánea de manejo de la falla cardíaca; de hecho, la mayoría de nuestros pacientes recibieron betabloqueadores, IECA/ARAll, e inhibidores de la aldosterona y un buen porcentaje tenían cardiorresincronizador con o sin desfibrilador.
Tabla 4 Hallazgos al examen físico, radiografía de tórax, ecocardiografía y paraclínicos de los pacientes con episodios de falla cardiaca aguda

\begin{tabular}{|c|c|}
\hline $\begin{array}{l}\text { Hallazgos al examen físico y } \\
\text { paraclínicos }\end{array}$ & $n(\%), n=143)$ \\
\hline \multicolumn{2}{|l|}{ Examen físico } \\
\hline Frecuencia cardíaca (latidos/min) * & $85(70-100)$ \\
\hline $\begin{array}{l}\text { Frecuencia respiratoria } \\
\text { (respiraciones } / \mathrm{min} \text { ) * }\end{array}$ & $20(18-23)$ \\
\hline Tensión arterial sistólica $(\mathrm{mm} \mathrm{Hg})^{*}$ & $112(97-137)$ \\
\hline Tensión arterial diastólica $(\mathrm{mm} \mathrm{Hg})^{*}$ & $70(60-81)$ \\
\hline Tensión arterial media $(\mathrm{mm} \mathrm{Hg})^{*}$ & $85(73,33-85,33)$ \\
\hline \multicolumn{2}{|l|}{ Saturación de oxígeno } \\
\hline$<70 \%$ & $4(2,80)$ \\
\hline $70-85 \%$ & $7(4,90)$ \\
\hline $85-92 \%$ & $20(13,99)$ \\
\hline$>92 \%$ & $111(77,62)$ \\
\hline Alteración del sensorio & $12(8,39)$ \\
\hline Ingurgitación yugular & $50(34,97)$ \\
\hline Crépitos pulmonares & $48(33,57)$ \\
\hline Galope S3 & $6(4,20)$ \\
\hline \multicolumn{2}{|l|}{ Edemas miembros inferiores } \\
\hline No & $53(37,06)$ \\
\hline Grado 1 & $31(21,68)$ \\
\hline Grado 2 & $42(29,37)$ \\
\hline Grado 3 & $15(10,49)$ \\
\hline Sin información & $2(1,40)$ \\
\hline Anasarca & $13(9,09)$ \\
\hline \multicolumn{2}{|l|}{ Hallazgos imagenológicos } \\
\hline Congestión venosa & $50(34,97)$ \\
\hline $\begin{array}{l}\text { Derrame pleural con edema } \\
\text { pulmonar }\end{array}$ & $24(16,78)$ \\
\hline $\begin{array}{l}\text { Derrame pleural sin edema } \\
\text { pulmonar }\end{array}$ & $28(19,58)$ \\
\hline Índice cardiotorácico $\geq 0,5$ & $92(64,34)$ \\
\hline \multicolumn{2}{|l|}{ Ecocardiograma } \\
\hline Fracción de eyección vi (\%)* & $30(16-55)$ \\
\hline VID $(m m)^{*}$ & $51(45-60)$ \\
\hline Insuficiencia mitral & $72(50,35)$ \\
\hline \multicolumn{2}{|l|}{ Hallazgos de laboratorio } \\
\hline Creatinina $(\mathrm{mg} / \mathrm{dl})^{*}$ & $1,25(0,9-1,7)$ \\
\hline BUN (mg/dl)* & $24,25(16,6-34)$ \\
\hline NT pro BNP * & $7797(3458-10500)$ \\
\hline Sodio* & $138(135-141)$ \\
\hline
\end{tabular}

VID: volumen de fin de diástole, vI: ventrículo izquierdo, BUN: nitrógeno ureico sanguíneo, NTproBNP: hormona N-terminal del péptido natriurético.

Las principales debilidades fueron la caracterización de los perfiles clínicos y hemodinámicos al momento del diagnóstico de la falla cardíaca descompensada, que no siempre sucedió al ingreso a urgencias sino que, en un grupo importante de pacientes, aconteció durante una hospitalización por causas distintas a falla cardiaca; además, durante ésta el cuadro clínico no siempre tuvo tendencia a la mejoría sino que en algunos empeoró y por tanto hubo cambio en la clasificación de Stevenson, lo cual explica el alto uso de inotrópicos intravenosos en este registro. 
Tabla 5 Resumen de estudios

\begin{tabular}{|c|c|c|c|c|}
\hline Estudio & Ospina et $\mathrm{al}^{15}$ & Castaño et $\mathrm{al}^{16}$ & Senior et $\mathrm{al}^{17}$ & Calderón et al \\
\hline Año publicación & 2004 & 2010 & 2011 & 2017 \\
\hline Duración del estudio & 1 año & 4 años & 6 meses & 7 meses \\
\hline$n$ & 218 & 370 & 106 & 99 \\
\hline Edad * & 68 & 69,5 & 62,4 & 63,57 \\
\hline Género masculino (\%) & 51,5 & 55,4 & 52,8 & 52,52 \\
\hline Etiología IC & NR & NR & Hipertensiva $45,2 \%$ & Isquémica $32,87 \%$ \\
\hline Comorbilidades & NR & NR & HTA, EPOC, ERC, DM & $\begin{array}{l}\text { HTA, DM, ERC, } \\
\text { valvulopatías }\end{array}$ \\
\hline NYHA & III $(63 \%)$ & ॥ (40\%) & III $(57,5 \%)$ & III O Iv $(79,02 \%)$ \\
\hline Perfil hemodinámico & NR & NR & Estadio B $(96,6 \%)$ & Estadio B $(55,07 \%)$ \\
\hline Causa de exacerbación & $\begin{array}{l}\text { No adherencia al } \\
\text { tratamiento }(50 \%)\end{array}$ & NR & NR & $\begin{array}{l}\text { Cirugía cardiaca } \\
(20,98 \%)\end{array}$ \\
\hline Estancia hospitalaria (días) * & 10 & NR & 11,42 & 6 \\
\hline FEVI (\%) * & 25 & NR & NR & 30 \\
\hline Disfunción sistólica (\%) & 73,2 & NR & 67,2 & 41,95 \\
\hline Medicamentos al ingreso & NR & $\begin{array}{l}\text { Betabloqueadores } \\
(16,8 \%) \\
\text { IECA }(87,6 \%) \\
\text { ARA II }(1,1 \%) \\
\text { Diurético ahorrador } \\
\text { de potasio }(50,5 \%) \\
\text { Diurético de asa } \\
(93,2 \%)\end{array}$ & $\begin{array}{l}\text { Betabloqueadores } \\
(32 \%) \\
\text { IECA }(73 \%) \\
\text { ARA II }(2 \%) \\
\text { Diurético ahorrador } \\
\text { de potasio }(34,9 \%) \\
\text { Diurético de asa (73\%) }\end{array}$ & $\begin{array}{l}\text { Betabloqueadores } \\
(62,9 \%) \\
\text { IECA o ARA ॥ }(66,4 \%) \\
\text { Diurético ahorrador } \\
\text { de potasio }(50,3 \%) \\
\text { Diurético de asa } \\
(64,3 \%)\end{array}$ \\
\hline Manejo de la falla cardiaca & $\begin{array}{l}\text { Diuréticos }(96 \%) \\
\text { Vasodilatadores } \\
(96 \%) \\
\text { Inotrópicos }(4 \%)\end{array}$ & NR & NR & $\begin{array}{l}\text { Diuréticos }(72 \%) \\
\text { Inotrópicos }(40,5 \%) \\
\text { Vasodilatadores } \\
(24,4 \%) \\
\text { Vasoactivos }(21,6 \%)\end{array}$ \\
\hline Mortalidad (\%) & 16,9 & NR & 0,9 & 12,12 \\
\hline
\end{tabular}

NR: no reportado, HTA: hipertensión arterial, EPOC: enfermedad pulmonar obstructiva crónica, ERC: enfermedad renal crónica, DM: diabetes mellitus, FEVI: fracción de eyección del ventrículo izquierdo, IECA: inhibidor de la enzima convertidora de angiotensina, ARA II: antagonista del receptor de angiotensina ॥, NYHA: clase funcional según la New York Heart Association, IC: insuficiencia cardiaca.

\section{Conclusiones}

La FCA descompensada es el perfil de presentación clínica más común en nuestra población seguido de edema pulmonar. El perfil hemodinámico más frecuente fue "caliente-húmedo"' (Stevenson B), resultados que concuerdan con estudios locales y registros internacionales. Nuestra mortalidad fue menor a la observada en otros estudios nacionales de FCA. Un tercio de las hospitalizaciones por FCA fueron en pacientes con FEVI preservada.

\section{Responsabilidades éticas}

Protección de personas y animales. Los autores declaran que para esta investigación no se han realizado experimentos en seres humanos ni en animales.

Confidencialidad de los datos. Los autores declaran que han seguido los protocolos de su centro de trabajo sobre la publicación de datos de pacientes.

Derecho a la privacidad y consentimiento informado. Los autores han obtenido el consentimiento informado de los pacientes y/o sujetos referidos en el artículo. Este documento obra en poder del autor de correspondencia

\section{Financiación}

Se contó con el patrocinio de la Fundación Valle del Lili y la Universidad Icesi.

\section{Conflicto de intereses}

Ninguno.

\section{Bibliografía}

1. Ponikowski P, Voors AA, Anker SD, Bueno H, Cleland JG, Coats AJ, et al. 2016 ESC Guidelines for the diagnosis and treatment of acute and chronic heart failure: The Task Force for the diagnosis and treatment of acute and chronic heart failure of the European Society of Cardiology (ESC). Developed with the special contribution of the Heart Failure Association (HFA) of the ESC. Eur J Heart Fail. 2016;18:891-975.

2. Nieminen MS, Brutsaert D, Dickstein K, Drexler H, Follath F, Harjola VP, et al. EuroHeart Failure Survey II (EHFS II): a survey on hospitalized acute heart failure patients: description of population. Eur Heart J. 2006;27:2725-36. 
3. Llorens P, Martín-Sánchez FJ, González-Armegol JJ, Herrero P, Jacob J, Alvárez AB, et al. Perfil clínico del paciente con insuficiencia aguda atendido en los servicios de urgencias: Datos preliminares del estudio EAHFE (Epidemiology Acute Heart Failure Emergency). Emergencias. 2008;20:154-63.

4. Gheorghiade M, Pang PS. Acute heart failure syndromes. J Am Coll Cardiol. 2009;53:557-73.

5. Mozaffarian D, Benjamin EJ, Go AS, Arnett DK, Blaha MJ, Cushman M, et al. Heart Disease and Stroke Statistics-2016 Update: A Report From the American Heart Association. Circulation. 2016;133:e38-60.

6. Barker WH, Mullooly JP, Getchell W. Changing incidence and survival for heart failure in a well-defined older population, 1970-1974 and 1990-1994. Circulation. 2006;113:799-805.

7. Jaramillo C, Gómez E, Hernandez E, Saldarriaga C, Flórez N, Buitrago $\mathrm{R}$, et al. Consenso colombiano para el diagnóstico y tratamiento de la Insuficiencia cardíaca crónica. Primera ed: Inversiones Tecnográficas S.A.S.; 2014.

8. Hernández-Leiva E. Epidemiology of acute coronary syndrome and heart failure in Latin America. Rev Esp Cardiol. 2011;64 Suppl 2:34-43.

9. Stevenson LW. Design of therapy for advanced heart failure. Eur J Heart Fail. 2005;7:323-31.

10. Nohria A, Tsang SW, Fang JC, Lewis EF, Jarcho JA, Mudge GH, et al. Clinical assessment identifies hemodynamic profiles that predict outcomes in patients admitted with heart failure. J Am Coll Cardiol. 2003;41:1797-804.

11. Jaramillo C, Gómez E, Edgar H, Saldarriaga C, Flórez N, Buitrago $\mathrm{R}$, et al. Consenso colombiano para el diagnóstico y tratamiento de la falla cardíaca aguda. Primera ed: Inversiones Tecnográficas S.A.S; 2014.

12. Januzzi JL, van Kimmenade R, Lainchbury J, Bayes-Genis A, Ordonez-Llanos J, Santalo-Bel M, et al. NT-proBNP testing for diagnosis and short-term prognosis in acute destabilized heart failure: an international pooled analysis of 1256 patients: the International Collaborative of NT-proBNP Study. Eur Heart J. 2006;27:330-7.

13. Dickstein K, Cohen-Solal A, Filippatos G, McMurray JJ, Ponikowski P, Poole-Wilson PA, et al. ESC guidelines for the diagnosis and treatment of acute and chronic heart failure 2008: the Task Force for the diagnosis and treatment of acute and chronic heart failure 2008 of the European Society of Cardiology. Developed in collaboration with the Heart Failure Association of the ESC (HFA) and endorsed by the European Society of Intensive Care Medicine (ESICM). Eur J Heart Fail. 2008;10:933-89.

14. Filippatos G, Farmakis D, Bistola V, Karavidas A, Mebazaa A, Maggioni AP, et al. Temporal trends in epidemiology, clinical presentation and management of acute heart failure: results from the Greek cohorts of the Acute Heart Failure Global Registry of Standard Treatment and the European Society of CardiologyHeart Failure pilot survey. Eur Heart J Acute Cardiovasc Care. 2014;3:1-7.

15. Ospina SAV, Gamarra HG. Características clínicas y epidemiológicas de la insuficiencia cardiaca en el hospital Universitario Ramón González Valencia de Bucaramanga, Colombia. Revista Salud UIS. 2004;36:125-31.
16. Castaño CJJ, Giraldo JF, Herrera HD, Jaramillo JJ, Noreña VJM, Restrepo M. Caracterización de los pacientes con insuficiencia cardíaca en el primer nivel de atención en la ciudad de Manizales (Colombia) 2005-2008. Archivos de Medicina (Col). 2010;10:127-38.

17. Senior JM, Saldarriaga C, Rendón JA. Descripción clínicoepidemiológica de los pacientes con falla cardíaca aguda que consultan al servicio de urgencias. Acta Med Colomb. 2011;36:125-9.

18. Spinar J, Parenica J, Vitovec J, Widimsky P, Linhart A, Fedorco $M$, et al. Baseline characteristics and hospital mortality in the Acute Heart Failure Database (AHEAD) Main registry. Crit Care. 2011;15:R291.

19. Antonelli M, Bonten M, Chastre J, Citerio G, Conti G, Curtis JR, et al. Year in review in Intensive Care Medicine 2011. II. Cardiovascular, infections, pneumonia and sepsis, critical care organization and outcome, education, ultrasonography, metabolism and coagulation. Intensive Care Med. 2012;38: 345-58.

20. Mebazaa A, Parissis J, Porcher R, Gayat E, Nikolaou M, Boas FV, et al. Short-term survival by treatment among patients hospitalized with acute heart failure: the global ALARM-HF registry using propensity scoring methods. Intensive Care Med. 2011;37:290-301.

21. Adams KF Jr, Fonarow GC, Emerman CL, LeJemtel TH, Costanzo MR, Abraham WT, et al. Characteristics and outcomes of patients hospitalized for heart failure in the United States: rationale, design, and preliminary observations from the first 100,000 cases in the Acute Decompensated Heart Failure National Registry (ADHERE). Am Heart J. 2005;149:209-16.

22. Yancy CW, Lopatin M, Stevenson LW, De Marco T, Fonarow GC, Investigators A.S.A.Ca. Clinical presentation, management, and in-hospital outcomes of patients admitted with acute decompensated heart failure with preserved systolic function: a report from the Acute Decompensated Heart Failure National Registry (ADHERE) Database. J Am Coll Cardiol. 2006;47:76-84.

23. Cleland JG, Swedberg K, Follath F, Komajda M, Cohen-Solal A, Aguilar JC, et al. The EuroHeart Failure survey programme a survey on the quality of care among patients with heart failure in Europe. Part 1: patient characteristics and diagnosis. Eur Heart J. 2003;24:442-63.

24. Komajda M, Follath F, Swedberg K, Cleland J, Aguilar JC, CohenSolal A, et al. The EuroHeart Failure Survey programme-a survey on the quality of care among patients with heart failure in Europe. Part 2: treatment. Eur Heart J. 2003;24:464-74.

25. Siirila-Waris K, Lassus J, Melin J, Peuhkurinen K, Nieminen MS, Harjola VP. Characteristics, outcomes, and predictors of 1-year mortality in patients hospitalized for acute heart failure. Eur Heart J. 2006;27:3011-7.

26. Fonarow GC, Abraham WT, Albert NM. Gattis Stough W, Gheorghiade $M$, Greenberg $\mathrm{BH}$, et al. Influence of a performance-improvement initiative on quality of care for patients hospitalized with heart failure: results of the Organized Program to Initiate Lifesaving Treatment in Hospitalized Patients With Heart Failure (OPTIMIZE-HF). Arch Intern Med. 2007;167:1493-502. 